\title{
SENTINEL MONITORING OF INFLUENZA
}

nfluenza monitoring began on June 4, 1990, with

collection of information on the patients of 24 participating general practitioners in the Sydney metropolitan area. This article outlines provisional information relating to the period June 4 to July 8 , 1990. The occurrence of influenza is based both on the general practitioner's clinical assessment of the patient having an influenza-like illness and on the application of defined criteria to the patient's symptoms.

All patients who present to participating GPs with an initial complaint of an influenza-like illness enter the study. The criteria for clinical diagnosis of influenza published in the International Classification of Health Problems in Primary Care, 2nd edition [ICHPPC-2 $]^{1}$ are then applied to the study subjects. 'Influenza-like illness' was deliberately not defined in the design of the study, but the ICHPPC-2 criteria are not applied to patients presenting with symptoms unrelated to acute respiratory tract infections. Subjects are classified as having influenza (as defined by ICHPPC-2) if at least four of the following eight criteria are fulfilled:

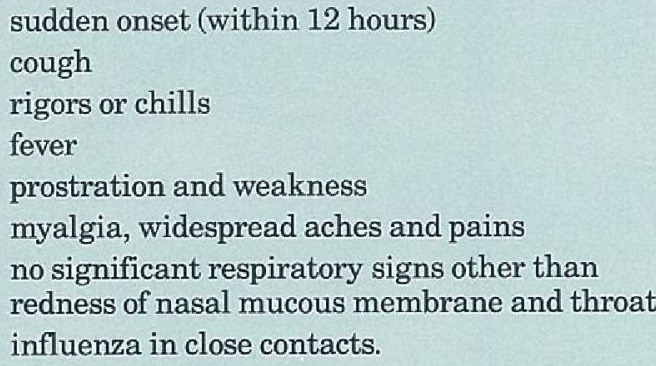

The proportions of all general-practitioner consultations that relate to both influenza-like illness and ICHPPC-2 defined influenza are shown in Figure 1. These proportions are expressed as the number of cases per 100 consultations.

\section{EDITORIAL COMMENT}

No previous studies of the incidence of influenza in the Sydney metropolitan area have been attempted. Therefore a comparison of the current incidence of influenza relative to previous periods is not possible. By July 16, 1990, information on 12,964 consultations had been processed. The proportion of generalpractitioner consultations relating to influenza-like illness is only $4.7 \%$, while the proportion fulfilling ICHPPC-2 criteria is $2.5 \%$.

Of the people presenting with influenza-like illness who had been immunised this year against influenza, $46 \%$ were below the NHMRC recommended age of 65 years. This does not take into account other medical conditions that could have made immunisation advisable.

Four-weekly reports of data collected in this study will appear in future issues of the Public Health Bulletin.

Wendy Manning RN BSCAgr, and Michael Levy MB BS MPH, Epidemiology and Health Services Evaluation Branch, Department of Health, NSW, and Mark Harris MB BS FRACGP $M D$, Department of Community Medicine, University of Sydney.

1. World Organization of National Colleges, Academies and Academic Associations of General Practitioners/Family Physicians (WONCA) Classification Committee. International classification of health problems in primary care, 2nd ed. Oxford University Press, Oxford, 1983.

\section{FIGURE 1}

\section{RATE OF INFLUENZA-LIKE ILLNESS}

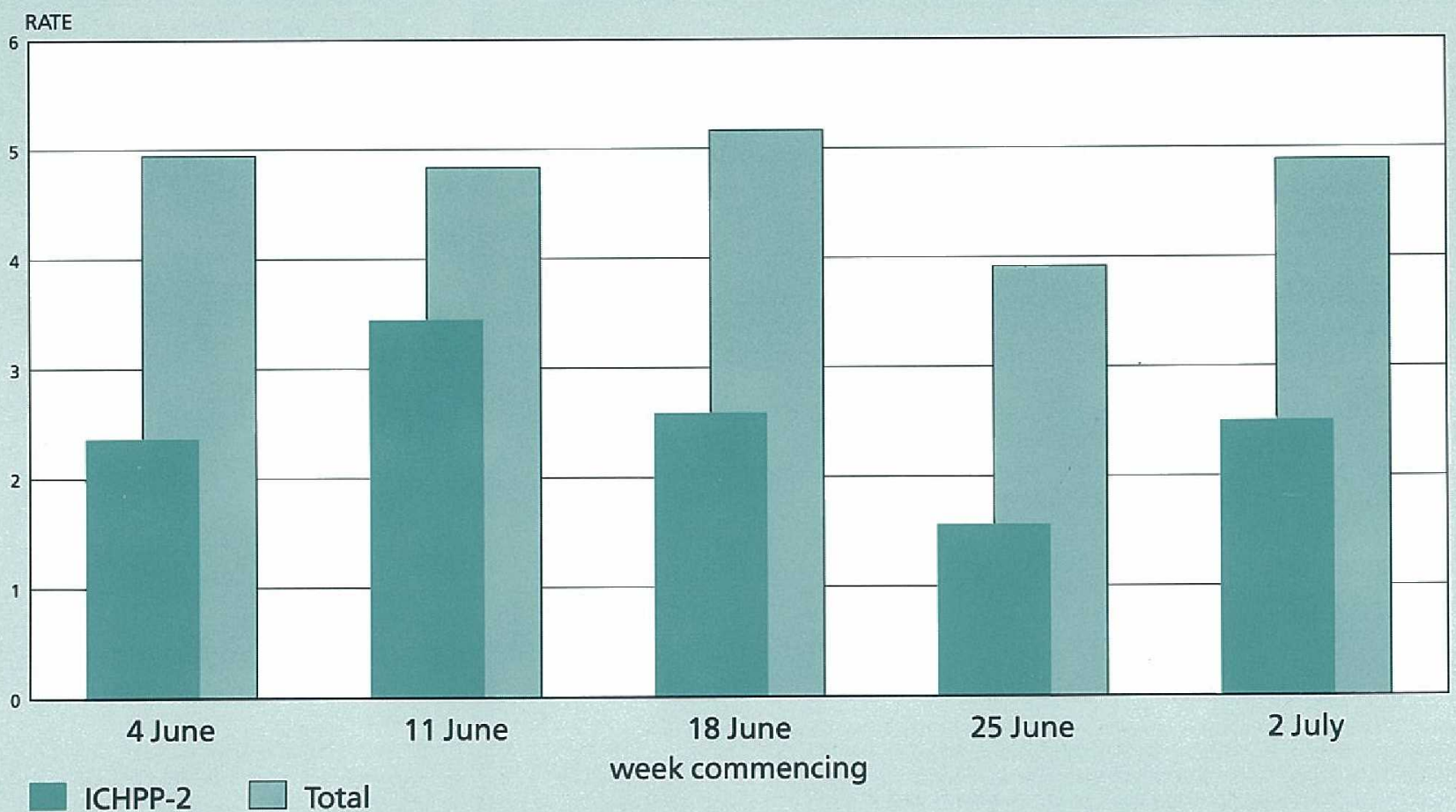

